\title{
Analysis of Water Application Techniques for Growing Tomatoes in Small Pots on Different Substrates. Note 2
}

\author{
Luigi Cavazza*, Antonia Patruno, Elio Cirillo \\ Dipartimento di Scienze e Tecnologie Agroambientali, Università di Bologna \\ Viale Fanin 44, 40127 Bologna Italy
}

Received: 5 August 2005. Accepted: 16 March 2006

\begin{abstract}
The response of the tomato plants to the irrigation regimes compared in the previous study, particularly when compared with the response to the peat fraction in the mixture, was modest, often irregular and subject to complex interactions with the former factor. This result could depend partially on the fact that the parameters for the water regime applied during the trials were chosen to prevent exposing the plants to excessive stress through lack of water or excess water. This note analyses the technique used to apply the water. Two main water application parameters were identified parameter $a$ expressing the mean moisture level of the pot during growing, and parameter $b$ expressing the amplitude of the variation in moisture level between the irrigation threshold and the level to which the water was topped up at each watering. The actual mean volume of irrigation during the cultivation period was compared with the theoretically predicted value and the discrepancy explained. The role of parameter $a$ and $b$ are proposed to define the irrigation regime applied and the water regime as such can be defined by these two parameters $a$ and $b$, as a general composite index. Furthermore the effect on the biological performance of both parameters $a$ and $b$ were studied obtaining a clearer picture of the effect of the water regime. The role of both parameters, $a$ and $b$ were synthesized in a specific composite index for each individual characteristic of the plant after taking in account the principal causes of their variations.
\end{abstract}

Key-words: indexes for irrigation regime, irrigation threshold, moisture content, top-up moisture.

\section{Introduction}

A previous study presented the experimental results obtained by comparing the development of tomato plantlets growing on different peatbased substrates with application of three different water regimes (Patruno et al., 2006). While the peat fraction in the mixtures played a predominant role not necessarily proportional to the mixture ratio (Wever, 1991, Burès et al., 1999) the influence of the water regime on the main characteristics of the plants was found to be modest. These results do not correspond to the results obtained by other Authors (Heiskånen, 1995; Sahin et al., 2002; Walczak et al., 2002) but possibly depend on the intervals between the "irrigation lower threshold point" and the "top-up" point being varied in harmony with variation of their mean. This note aims at investigating the effects of the three water regimes analysing their individual components and trying to give a better understanding of the irrigation effect.

\section{Materials and methods}

The comparison was carried out between four mixtures of peat with sand and the same number with perlite with the general characteristics indicated in Table 1 and in more detail in Patruno et al. (in this review). Tomatoes were cultivated in pots up to a height of about $10-15 \mathrm{~cm}$, irrigating according to the criteria described in more detail in the previous study. The water in the various pots was monitored by weighing them each day on a balance. As soon as the water content dropped below the established irrigation lower threshold, it was brought up to the established top-up threshold and the amount of

\footnotetext{
* Corresponding Author: Tel.: +39 051 2096678; Fax: +39 051 2096241. E-mail address: lcavazza@agrsci.unibo.it
} 
Table. 1 Hydrological parameters for water application.

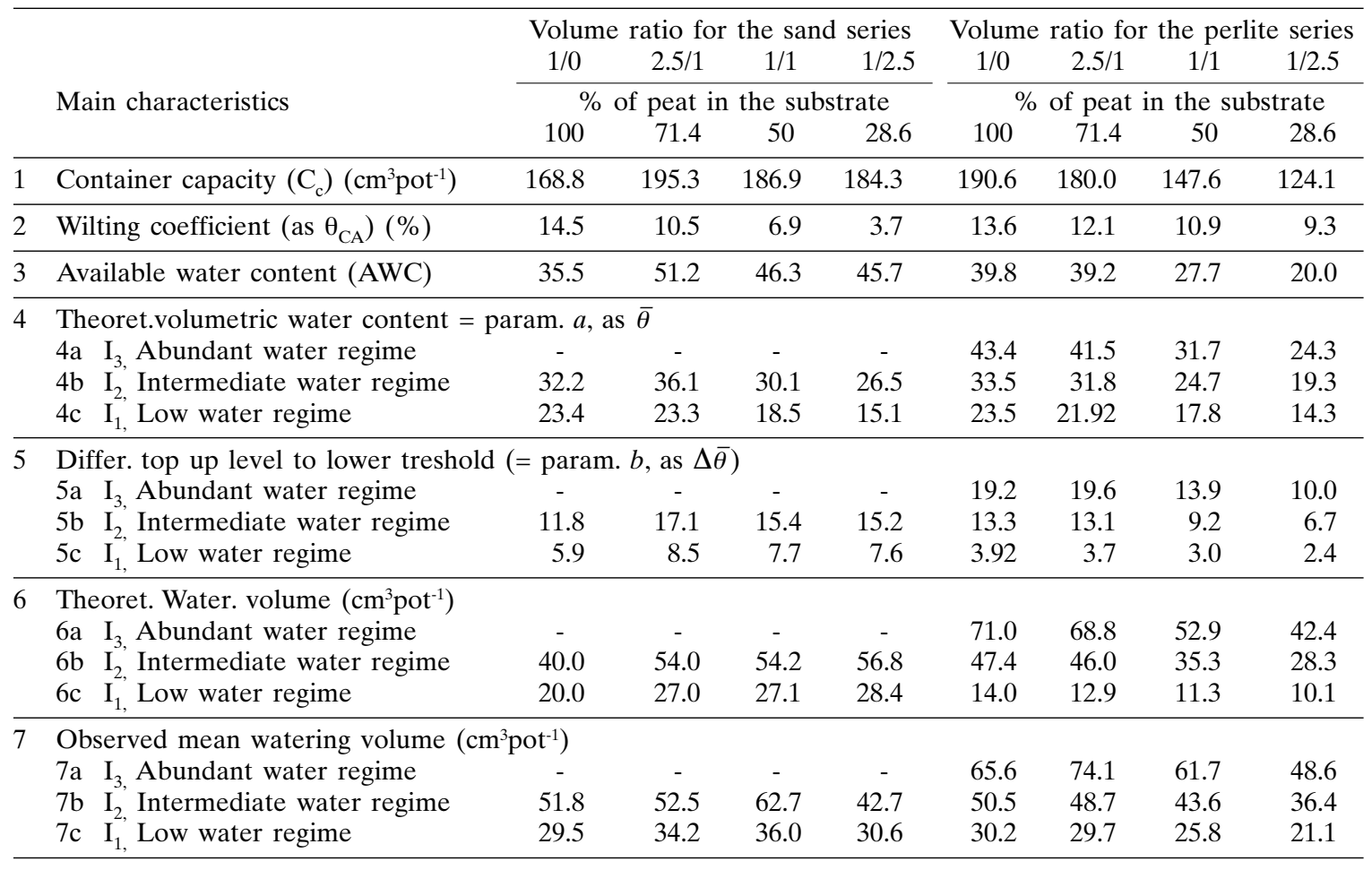

water added was measured.. This work is limited to an examination of the mean data of the following series: one of peat-sand mixtures without fertiliser under two water regimes with two replications, and one series of peat-perlite mixtures fertilised and grown under three water regimes with two replications. The data from a total of 40 pots (16 in the peat-sand series and 24 in the peat-perlite series) were considered here.

For the statistical analysis of the data in the previous paper, each of the three water regimes were considered as a separate complex treatment with the result that many of the effects of the single technique of water application on certain traits of the plants appeared masked and slight or not significant. More precisely, the technique of water application in this trial can be analysed in two components: one, which we shall indicate as parameter $a$, expresses the average moisture content at which the soil was kept during the growing period and is given by the mean of the irrigation "top-up threshold" value and the "lower irrigation threshold" value, and second component, the parameter $b$, which is the difference between the "top-up moisture threshold" and the "irrigation lower threshold", and expresses the amplitude of the range of moisture levels to which the plant was exposed within those limits. Through a traditional analysis of variance it was not possible to distinguish between the role of those two independent ${ }^{1}$ parameters on the basis of which the three water regimes were programmed.

The calculations used to identify parameters $a$ and $b$, expressed as the volumetric moisture content for each pot, are given in detail in the previous paper and the essentials are given in Table 1.

\section{Discussion}

Let start considering both parameters, $a$ and $b$, applied simultaneously. Note that each of this taken to its respective extreme ( 0 and $100 \%$ for both $\bar{\theta}=a$ and for $\Delta \theta=b$ ) can be expected to produce lethal or sub lethal effects on the crops.

\footnotetext{
1 The two threshold values as volumetric contents, $\theta_{u}$ and $\theta_{1}$, give 2 degrees of freedom; one of these is taken up for their mean (parameter a) and the second expresses independently their deviance (parameter b), Dixon and Massey 1951.
} 
This suggested that their mathematical product could possibly represent an index of the water regime, independently of its consequences on the biological effects. Thus the plan for the irrigation regime can be described by a generic composite index of the water regime, in particular we can use the product of $a \times b$ which is strictly equivalent to:

$$
\left.\left[\theta_{\mathrm{u}}+\theta_{1}\right) / 2\right] \times\left(\theta_{\mathrm{u}}-\theta_{1}\right)=\left(\theta_{\mathrm{u}}{ }^{2}-\theta_{\mathrm{l}}{ }^{2}\right) / 2
$$

where the subscript $u$ and $l$ indicate "upper" and "lower", irrigation threshold.

However, this index is not able to give information on the possible effects and relative weight which the two parameters may have on different traits of the plants when the irrigation is applied and the plants are growing.

The above considerations show that each biological manifestation (dry weight of plants, leaf area, water consumption per pot, etc.) of each plant species can be affected by all different factors tested in an experiment and in the case of irrigation, this can be splitted into two components $a$ and $b$. Thus in our experiment firstly a comparison can be made between the observed mean volumes of irrigation per pot obtained dividing the observed total water consumption for treatment (Table 2 of Note 1) by the actual number of irrigations performed during the growing period (not given in detail; it depends from the plant growth in each pot). The results obtained can be seen on line 7a,b,c of Table 1 . A theoretical estimate of the same irrigation volumes can, on the other hand, be obtained from parameter $b$, taken as the basis for the water treatment applied. Each individual water application is, in theory, equal to $b$ (expressed in terms of volumetric moisture) multiplied by the volume of soil in each pot (Table 1 of Note 1). The results are given on line $6 a, b, c$ of Table 1 .

The value for observed applications are higher than theoretical levels. Apart from minor inevitable experimental errors, the differences (not reported for the sake of brevity) can be justified by the following considerations. Firstly, at the time of water applications (the same time each day), a period no longer than 24 hours could have passed since the previous day's measurement, a period during which evaporation and transpiration had continued to take place. It can therefore be expected that for each pot the loss of water in the meantime will be approximately half the mean daily evaporationtranspiration during the whole trial (water consumption divided by the length of the trial). On the other hand, this mean loss due to evaporation-transpiration occurred only at each water application and must therefore be proportional to the number of waterings (for example, greater for regime $\mathrm{I}_{1}$ ) and inversely proportional to the effective mean volume of irrigation (greater for regime $\mathrm{I}_{3}$ ) for each pot. The differences between lines $7 \mathrm{a}, \mathrm{b}, \mathrm{c}$, and lines $6 \mathrm{a}, \mathrm{b}, \mathrm{c}$, are in fact extremely irregular but their mean magnitude is in agreement with the above. The mean values for each combination of water regimes per substrate series are, in fact, for sand $\mathrm{I}_{1}=+6.95$ and $\mathrm{I}_{2}=+1.18$ and for perlite $\mathrm{I}_{1}=$ +14.63 and $I_{2}=+5.55$ and $I_{3}=+5.46$, in other words, becoming greater as the values of parameter $b$ become smaller. The greatest discrepancy between observed and theoretical volumes can be expected in regime $\mathrm{I}_{3}$ (the highest mean values obtained with the perlite; it also depend on the albeit slight differences of fertilization etc.).

The data on the dry weight of the plants (the peat effect is significant) and on leaf area (Figure $1 a-b$; the effects of peat, water regimes and their interaction are significant) for the peatsand series not just confirm the importance of the peat fraction in the mixture (already described in the previous paper) but also suggests an interesting and relatively regular increase in the slope of these segments as the peat fraction increases. This means an increase in the efficiency of the mean theoretical volumetric moisture (this efficiency can be expressed as the $d y / d x$ derivative, where $y$ is the biological response of the plants and $x$ the moisture level considered, in other words, parameter $a$ ).

The case of the peat-perlite mixtures for which data are available for three water regimes is more complex. The corresponding graphs (Figure 1c-d; the effects of the peat and, for the dry matter, the water regime are significant), confirm the role of the percentage of peat, but clearly demonstrate that as parameter $a$ increases, there is a tendency for the biological response to show an optimal intermediate level, dropping slightly at the highest moisture levels or extending in an asymptotic curve in the case of the leaf area (1d). The contrast of the points 

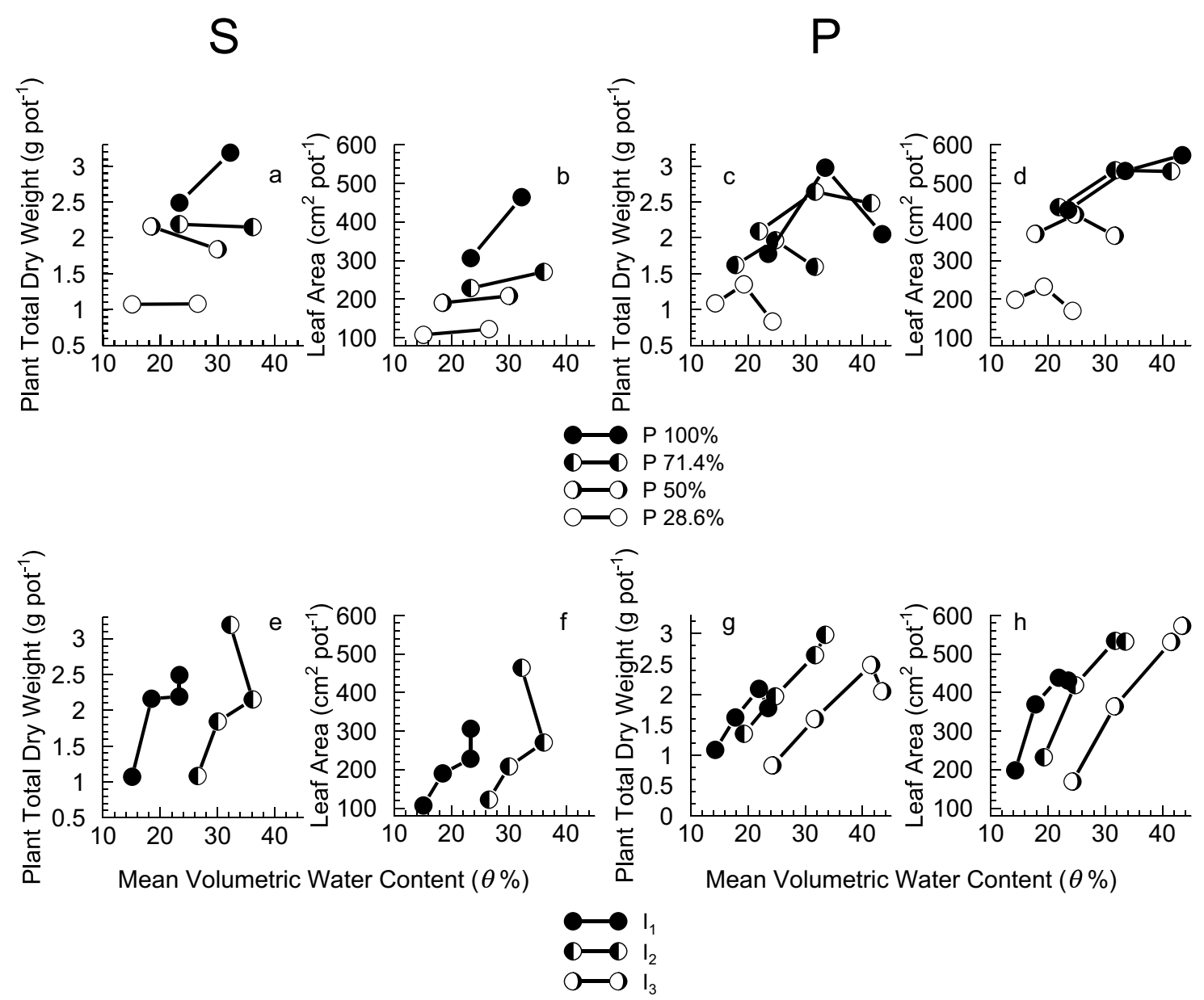

Figure 1. Effect of the mean volumetric water content (param. $A=\bar{\theta}$ ) on plant total dry weight and leaf area of tomato grown in different substrates of peat and sand $(\mathrm{S})$ or peat and Perlite $(\mathrm{P})$. The proportion of the peat in the mixtures are in volume $100 \%, 71.4 \%, 50 \%, 28.6 \%$. The effect of the irrigation regimes are presented in the lower graphs; these are definited by mean volumetric water content (abscissas) as well as different excursion moisture (parameter $b$, see table 1)

at $100 \%$ peat between leaf area and dry weight of the plants suggest a higher water content in the tissues at the highest values of $a$.

In Figure 1, the same data were used to construct in the corresponding bottom sections curves referring to a given water regime. In this graph, the uppermost point of each curve corresponds to $100 \%$ peat and the opposite point to the minimum fraction of peat in the mixture. These curves show a marked tendency to parallel alignment, clearly illustrating the role of the peat fraction in the mixture. It is evident that the same mean quantity (dry weight or leaf area) can be obtained with very different water regimes when changing the peat ratio.

It seems reasonable to assume that each biological effect $(B)$ depends on: (a) given gener- al traits (for example, leaf area, given species); (b) growing conditions for group of treatments (such as sand or perlite series, fertilized or not); (c) parameters of individual pot (e.g. the container capacity, $C_{\mathrm{c}}$ ); (d) parameters $a$ and $b$, for irrigation. To analyze these effects one would choose appropriate functions. In our case it seemed better to choose as a suitable function the following logarithmic equation (which allows for multiplicative relationships between the factors mentioned above):

(2) $\ln B=\mathrm{b}_{0}+\mathrm{b}_{1} \ln { }_{C \mathrm{p}}+\mathrm{b}_{2} \ln C_{\mathrm{c}}+\mathrm{b}_{3} \ln a+\mathrm{b}_{4} \ln b$

in which $B=$ biological performance (production of dry matter, leaf area etc); $c_{\mathrm{p}}=$ the distinctive characteristic of each group (e.g. sand and perlite series, fertilized or not a slightly dif- 


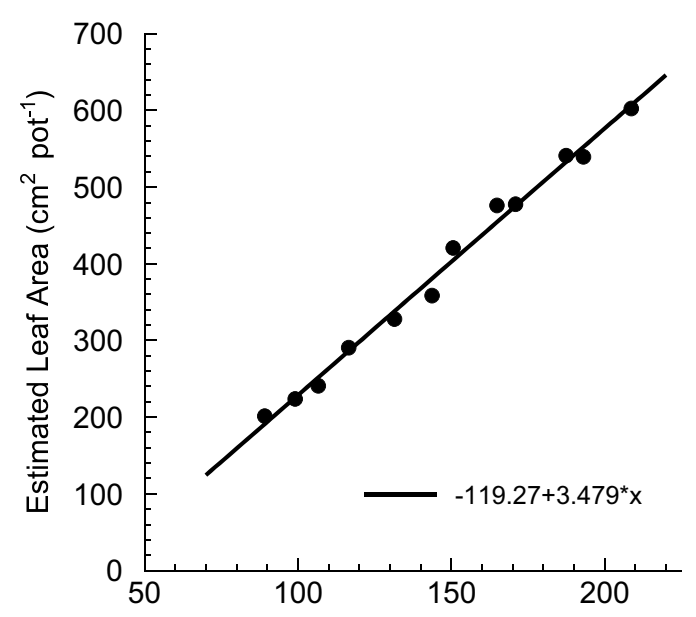

Specific Composite Index of Water Regime

Figure 2. Relationship between estimated leaf area and specific composite index of water regime.

ferent temperature etc.); $C_{\mathrm{c}}$ typical characteristics of each pot and $a$ and $b$ as the irrigation parameters previously evaluated. The b's in equation 2 are the coefficients of partial regression; $b_{0}$ represents all the factors not identified in addition to the above. The above multiple regression equation was tested by using all 40 pots in the trial (sand and perlite), using contrasting indexes $c_{\mathrm{p}}=$ 1 for the sand and 2 for the perlite.

The above equation applied to the values for leaf area of the two trials considered together gave excellent results with a significance of $0.001 \mathrm{P}$ for all four causes of variation considered (the coefficients $b_{3}$ and $b_{4}$ were also highly significant at $0.001 \mathrm{P})$. For leaf area, $\left(A_{\mathrm{f}}\right)$, the result is therefore:

$$
\ln \left(A_{\mathrm{f}}\right)=-2.302+0.508 \ln c_{\mathrm{p}}+
$$

$$
0.573 \ln C_{\mathrm{c}}+1.946 \ln a-0.668 \ln b
$$

with $\mathrm{R}^{2}=0.75996$

From $3 a)$, the following is obtained:

$$
\mathrm{A}_{f}=\frac{c_{p}^{0.508}}{e^{2.302}} \times \mathrm{C}_{c}^{0.573} \times \frac{\mathrm{a}^{1.946}}{\mathrm{~b}^{0.668}}
$$

in which the last fraction expresses the specific composite index of the water regime as a single numerical value expressing the effect of the overall water regime applied, with reference to the characteristic $\left(A_{\mathrm{f}}\right)$ for which the effect is inferred.

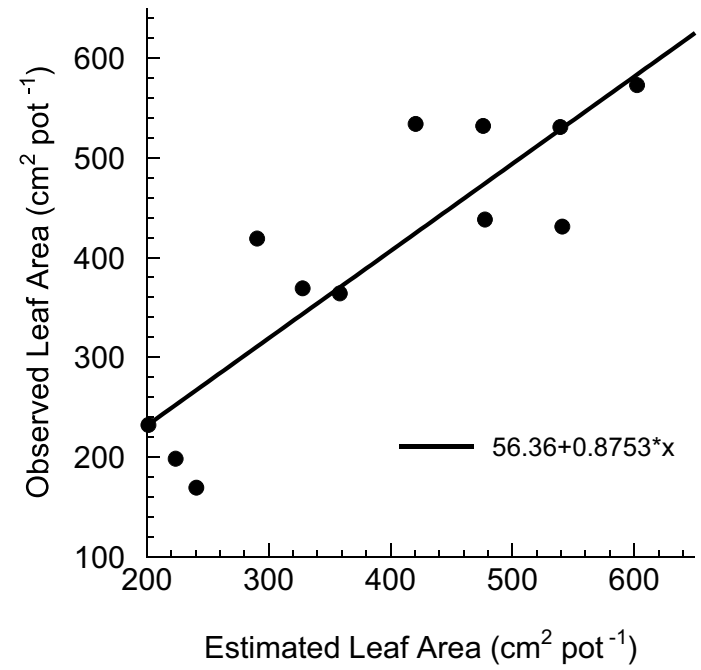

Figure 3. Relationship between estimated and observed leaf area using equation $3 \mathrm{~b}$. Note the correspondence of deviations to details in Figure 1e,d.

In this example, formula 3a was also applied to only the leaf area of the peat-perlite trial taking $\mathrm{c}_{\mathrm{p}}=2$ (peat-perlite), the values of $C_{\mathrm{c}}$ from the 1st line on the right of tab.1 and the values of parameters $a$ and $b$ from the lines 4a,b,c and $5 a, b, c$ of the same table. The result shown in Figure 2 representing the estimated leaf area on the basis of the specific composite index relating to the water regime (the small discrepancies from straight line can be attributed to random differences in container capacity). The relationship between observed leaf area and estimated leaf area is shown (Figure 3). In this case the points are poorly aligned, in accordance with a not to high value of 0.76 found for $\mathrm{R}^{2}$ (despite the very high significance of the single effects considered). This suggests that some relevant effects were not yet taken in account by equation 2. Indeed traces can be found in Figure 3 which compared to Figure 2 corresponds to the tendency in the perlite series for the existence of an optimal value of the parameter $a$ differing for each peat level. It seems that these interactions escaped in the considerations leading to equation 2 .

\section{Conclusions}

The initial examination of the data as given in the previous paper identified the effect of the 
water regimes applied en bloc and so escaped to analyze the components of the irrigation programs. On the basis of these, a generic composite index for the water regime can be defined in terms of the irrigation top-up threshold level and the lower threshold moisture level (eq. 1) when programming the irrigation. If account is also required for various manifestations of a single crop (water consumption in single pots, dry weight, leaf area, etc.) or of different crops, these effects can be analysed separately, assuming adequate functions (e.g. a logarithmic one) to obtain an experimentally determined specific composite index of the water regime referred to the effect considered (eq. 3b). So, for example, the results obtained by multiple regression analysis of leaf area expansion data clearly showed (with very high significance, all at $0.001 \mathrm{P}$ ) the possibility of separating general effects from single pot effects as the container capacity as well as the mean moisture level at which the plants were kept in the various water regimes (parameter $a$ ) and the range of moisture levels to which the plants were subjected between the "upper irrigation threshold" and the lower threshold (parameter $b$ ). With reference to this regression equation a single specific com- posite index for the water regime expresses the combined effect of parameters $a$ and $b$.

\section{References}

Patruno A., Cavazza L., Cirillo E. 2006. Growing tomato plantlets on various mixtures of peat and sand or peat and perlite. Note 1. Ital. J. Agron, 2:315-321.

Cavazza L., Patruno A., Cirillo E. 2005. An approximate indirect evaluation of water retention relationship for small pots. Adv. Hort. Sci., 19:111-115.

Dixon W.J., Massey F.J.V. 1951. Introduction to statistical analysis. Mc Graw-Hill. New York.

Burès S., Martinez F.X., Pera N. 1999. Physical properties of substrate mixture according to the characteristic of the original materials. Acta Horticulturae, 209:207-214.

Heiskåen J. 1995. Physical properties of two-component growth media based on Sphagnum peat and their implications for plant available water and aeration. Plant and Soil, 172:45-54.

Sahin U., Anapali O., Ercisli S. 2002. Physicochemical and physical properties of some substrates used in horticulture. Gartenbauwissenschaft, 67:55-60.

Walczak R., Rovdan E., Withowska-Walczok B. 2002. Water retention characteristic of peat and sand mixtures. Internat. Agrophysics, 16:161-165.

Wever C. 1991. Guide values for physical properties of peat substrates. Acta Horticulturae, 294:41-47. 